\section{Nanoscopy in a Living Mouse Brain}

\author{
Sebastian Berning, ${ }^{1}$ Katrin I. Willig, ${ }^{1 *}$ Heinz Steffens, ${ }^{1}$ Payam Dibaj, ${ }^{2}$ Stefan W. Hell ${ }^{1 *}$
}

$\mathrm{C}$ ells, the functional units of life, are best studied in vivo. This is particularly true for neurons, which perform their basic function of information processing by connecting with their neighbors. Therefore, unraveling the inner workings of the brain requires the imaging of neurons in the living animal. Although confocal and multiphoton microscopy can visualize neurons tagged with fluorescent proteins in transgenic living systems, they cannot discern features closer than half the wavelength of light (200 to $300 \mathrm{~nm})(1)$. By causing such features to fluoresce sequentially, stimulated emission depletion (STED) microscopy and other emerging superresolution techniques have now overcome this barrier (2). Whereas these techniques have been applied throughout the life sciences, in vivo nanoscale imaging of cells in higher animals has remained elusive. We used STED microscopy to superresolve neurons and their subtle dynamics in the cerebral cortex of a living mouse.

We developed an upright scanning STED fluorescence microscope with a 1.3-numerical aperture (NA) lens focusing a $80-\mathrm{MHz}$ train of 70-ps excitation pulses of 488-nm wavelength on the animal brain (Fig. 1A). To visualize neurons, we used heterozygous $\operatorname{TgN}$ (Thy1-EYFP) mice expressing enhanced yellow fluorescent protein (EYFP) as a nonfusion protein in neuronal cytoplasm (3), which is under the control of the regulatory element from the thyl gene. The focused excitation pulses were coaligned and synchronized with doughnut-shaped 592-nm STED pulses of 300-ps duration and $25-\mathrm{mW}$ focal power for silencing the EYFP. The fluorescence was imaged onto a confocal detector so that 600 -nm-thick layers inside the brain could
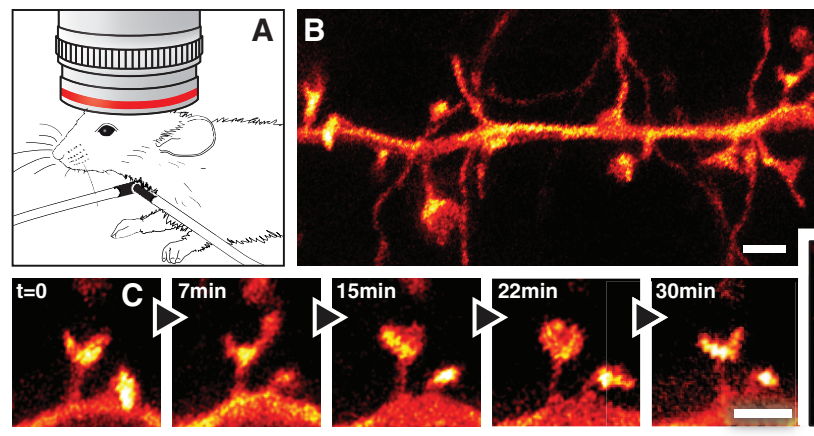

be discriminated. Optical access was provided by a cover glass-sealed hole in the skull, exposing the mouse's somatosensory cortex. The anaesthetized mouse was artificially ventilated and controlled for its vital functions, such as body temperature, ventilation, blood oxygenation, and heart function [by recording the electrocardiogram]. Rigid construction and keeping the optical paths short protected our setup from external vibrations. Thus, the images could be recorded without active vibration compensation or numerical image processing. Cardiovascular and respiratory motion was suppressed by an optimized surgical preparation procedure, which was particularly important for recording potential movements of the dendritic spines. Although such movements have been observed in hippocampal organotypical slices of 5- to 7-day-old mice $(4,5)$ and during de novo growth in the developing cortex (6), until now it has remained unclear whether they can occur in the adult animal brain.

The STED image in Fig. 1B shows a dendritic process within the molecular layer of the somatosensory cortex of a $\operatorname{TgN}$ (Thyl-EYFP) mouse, located 10 to $15 \mu \mathrm{m}$ below the surface. The STED images show structures of $<70 \mathrm{~nm}$ in size (Fig. 1D), indicating that the resolution is at least of that order. Recording images every 7 to $8 \mathrm{~min}$ revealed that adult dendritic spines can undergo morphologic changes and movements (Fig. 1C, 0 to $30 \mathrm{~min}$, and movie S1) on the time scale of minutes. These movements were repeatedly observed in all six individuals imaged. The mice were aged between 66 and 205 days. To exclude random defocus from being mistaken for movement, we rendered each image by a maximum intensity projection of a stack of five
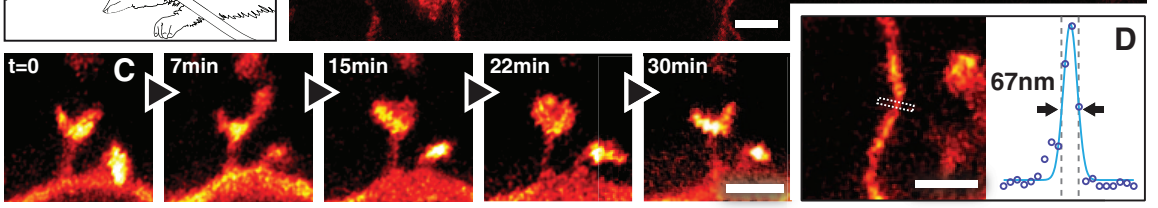

Fig. 1. STED microscopy in the molecular layer of the somatosensory cortex of a mouse with EYFP-labeled neurons. (A) Anesthetized mouse under the objective lens (63x, NA 1.3, glycerol immersion) with tracheal tube. (B) Projected volumes of dendritic and axonal structures reveal (C) temporal dynamics of spine morphology with (D) an approximately fourfold improved resolution compared with diffraction-limited imaging. Curve is a three-pixel-wide line profile fitted to raw data with a Gaussian. Scale bars, $1 \mu \mathrm{m}$. images with 600-nm-depth spacing. Although the dendrite of origin retained largely the same shape throughout the experiments, morphological changes were found at the head and neck regions of the dendritic spines, potentially reflecting alterations in the connectivity of the neural network, as is seen in the immature brain. Apparently, dendritic spines can alter their morphology in the intact somatosensory cortex of the adult mouse.

Although absorption of the 592-nm STED beam by EYFP is negligible, adverse absorption in the tissue may occur at this wavelength. Indeed, slight local swellings have occasionally been observed for relatively thick dendritic processes featuring many mitochondria. However, we did not observe degradation or disaggregation of dendritic processes as known to occur right after cell death or exitus. A remedy should be the use of red fluorescent proteins calling for STED beams of wavelengths $>700 \mathrm{~nm}$, where absorption by mitochondrial metabolites is negligible. Another option is to use transgenic animals tagged with reversibly switchable fluorescent proteins, enabling the STED-like nanoscopy called RESOLFT (reversible saturable optical fluorescence transitions) that requires far lower light intensities than STED (7). Future long-term in vivo studies should solve central aspects of brain development and, using mouse models, also of brain disease. Altogether, we expect in vivo optical nanoscopy to assume a major role in the quest for deciphering our primary organ.

\section{References and Notes}

1. T. Misgeld, M. Kerschensteiner, F. M. Bareyre, R. W. Burgess, J. W. Lichtman, Nat. Methods 4, 559 (2007).

2. S. W. Hell, Science 316, 1153 (2007).

3. G. P. Feng et al., Neuron 28, 41 (2000).

4. U. V. Nägerl, K. I. Willig, B. Hein, S. W. Hell, T. Bonhoeffer, Proc. Natl. Acad. Sci. U.S.A. 105, 18982 (2008).

5. N. T. Urban, K. I. Willig, S. W. Hell, U. V. Nägerl, Biophys. J. 101, 1277 (2011).

6. H. B. Kwon, B. L. Sabatini, Nature 474, 100 (2011).

7. T. Grotjohann et al., Nature 478, 204 (2011).

Acknowledgments: The experiments were performed according to the ethics guidelines of national law regarding animal protection procedures and were authorized by the ethics committee of the Max Planck Institute for Biophysical Chemistry and by the responsible authorities, the Niedersächsisches Landesamt für Verbraucherschutz. We thank A. Schönle for the software Imspector. S.W.H. holds patents on STED microscopy: German patent DE 4416558 C2 and U.S. Patent US5731588A.

\section{Supporting Online Material}

www.sciencemag.org/cgi/content/full/335/6068/551/DC1 Materials and Methods

Figs. S1 and S2

Movie S1

17 October 2011; accepted 15 December 2011 10.1126/science.1215369

${ }^{1}$ Department of NanoBiophotonics, Max Planck Institute (MPI) for Biophysical Chemistry, Am Fassberg 11, 37077 Göttingen, Germany. ${ }^{2} \mathrm{MPI}$ for Experimental Medicine, Hermann-ReinStrasse 3, 37075 Göttingen, Germany.

*To whom correspondence should be addressed. E-mail: kwillig@gwdg.de (K.I.W.); shell@gwdg.de (S.W.H.) 